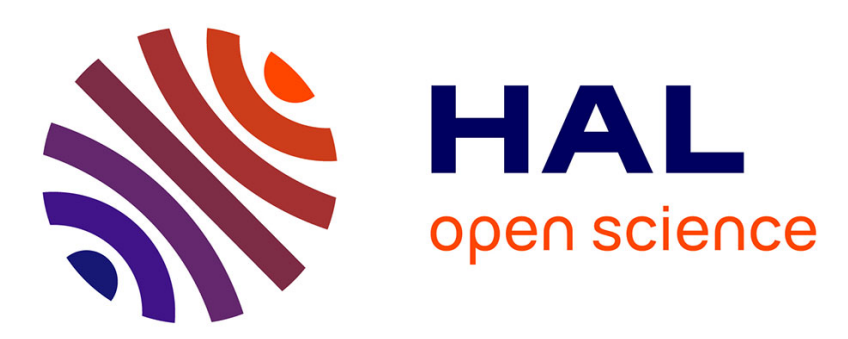

\title{
A hybrid lipid oligonucleotide: a versatile tool for supramolecular chemistry
}

Arnaud Gissot, Khalid Oumzil, Amit Patwa, Philippe Barthélémy

\section{To cite this version:}

Arnaud Gissot, Khalid Oumzil, Amit Patwa, Philippe Barthélémy. A hybrid lipid oligonucleotide: a versatile tool for supramolecular chemistry. New Journal of Chemistry, 2014, 38 (11), pp.5129-5134. 10.1039/C4NJ00850B . hal-02484571

\section{HAL Id: hal-02484571 https://hal.science/hal-02484571}

Submitted on 24 Feb 2020

HAL is a multi-disciplinary open access archive for the deposit and dissemination of scientific research documents, whether they are published or not. The documents may come from teaching and research institutions in France or abroad, or from public or private research centers.
L'archive ouverte pluridisciplinaire HAL, est destinée au dépôt et à la diffusion de documents scientifiques de niveau recherche, publiés ou non, émanant des établissements d'enseignement et de recherche français ou étrangers, des laboratoires publics ou privés. 


\title{
Hybrid Lipid Oligonucleotide: a versatile tool for supramolecular chemistry
}

\author{
Arnaud Gissot, ${ }^{\mathrm{a}, \mathrm{b}}$ Khalid Oumzil, ${ }^{\mathrm{a}, \mathrm{b}}$ Amit Patwa, ${ }^{\mathrm{a}, \mathrm{b}}$ and Philippe Barthélémy ${ }^{\mathrm{a}, \mathrm{b}, *}$
}

Lipid Oligonucleotides (LONs) are emerging as promising supramolecular tools for biomedical and technological applications. In this contribution we highlight recent advances in the area of LONs with an emphasis on their supramolecular properties and applications. In the first section we focus on the design, self-assemblies and applications of LONs. In the last section, we describe recent biomedical applications thereof.

\section{Introduction}

Nucleic acids and lipids are central molecules in living organisms. While the former is responsible for the storing and propagation of genetic information, the latter allow for the protection and compartmentalization of the living matter from the external environment. The chemical combination of a lipid with an oligonucleotide, the so-called Lipid OligoNucleotides (LONs), have been realized with the aim of combining and optimizing the properties of both biomolecules. ${ }^{1}$ In fact, the propensity of the lipidic segment of the LONs to aggregate in water brings about new self-assembling opportunities to these bio-inspired amphiphiles. LONs can therefore self-assemble into micelles, ${ }^{2}$ liposomes ${ }^{3}$ and nanoparticles. This phenomenon is expected to positively impact the biological effect of the nucleic acid segment of the LON. These different fields have already been covered by us or others in the past. ${ }^{1,4,5}$ This contribution will therefore mainly focus on the recent advances with an emphasis on supramolecular and biomedical applications.

\section{Lipid-oligonucleotide conjugates, from molecular to supramolecular self-assemblies}

Many biomolecules have been covalently attached to nucleic acid sequences to impart new properties to the oligonucleotides e.g. targeting properties (RGD peptides, carbohydrates), higher affinities for their complementary sequence (oligospermine in zip nucleic acids), ${ }^{6}$ or cell membrane crossing capabilities with lipid conjugates. Recent developments in the field of oligonucleotide conjugates have been reviewed lately. ${ }^{7-9}$ Lipid conjugation to ONs was primarily thought of as a mean to anchor the ON to cell membranes, ultimately leading to the internalization of the $\mathrm{ON}$ sequence. Many different lipidantisense $\mathrm{ON}$ have consequently been synthesized in the past. Basically there are three different positions for lipid conjugation to ONs: (i) 3'-terminal, (ii) 5'-terminal and (iii) intra-chain position. At each location, the lipid conjugates can be incorporated at various sites i.e. at the phosphate backbone, at the sugar (at 2'-, 3'-, 5'-positions, etc.) and/or at the nucleobase. The specific site(s) of lipid conjugation has/have an impact on the biological and physical properties of the LONs.
Therefore, depending on its application, the conjugation site(s) has/have to be carefully chosen so that it facilitates the function of LONs without compromising its binding properties (usually hybridization with the complementary ONs). The synthetic methods that have been used for the preparation of LONs have been thoroughly reviewed and will not be covered here. ${ }^{1,5,10}$

\subsection{Supramolecular assemblies}

The presence of a lipid in ON sequences improves their solubility in organic solvents. If only one lipid does not seem to be sufficient to provide full solubility, a TG 4 T DNA sequence with dodecyl chains at each phosphotriester linkage is soluble in chloroform or THF. ${ }^{11}$ Interestingly, this minimal sequence prone to form G4-quadruplexe in water kept the ability to form this secondary structure motif and extract alkali metals in chloroform or THF. Wang et al. took profit of the higher tolerance of LONs toward organic co-solvents to play around the solvent composition to trigger the switch between different supramolecular architectures. ${ }^{12}$ While micellar aggregates are formed in the presence of THF, their hybrid DNA-dendron LON formed nanofibers after gently evaporating THF followed by slow cooling. The authors hypothesized that the organic cosolvent eliminated $\pi-\pi$ stacking interactions important for the integrity of the nanofibers.

Nevertheless, ONs and LONs are overwhelmingly used in water. Here, the lipidic tether brings about amphiphilic character to the ON. Yet, unlike readily available conventional amphiphiles whose self-aggregation properties have been extensively studied over the years, little is still known about the nature, the thermodynamic and kinetic parameters of LON aggregates. In fact, the solid phase synthesis of $\mathrm{ON}$ is the method of choice for the production of LONs (and more generally ONs). ON syntheses are conveniently carried out at the 0.2 up to a few dozen $\mu$ mole scale at best at the laboratory level. These quantities clearly limit the wealth of physicochemical experiences that can be carried out with LONs. As an example, parameters as basic as the critical aggregation concentrations (cac) of LONs are rare in the literature. Besides, these values are usually obtained with hydrophobic fluorescence probes, which can sense polarity change in their environment. With the notable exceptions of LONs with either 
a cholesterol, or short consecutive alkyl chains at the internucleosidic phosphorus, ${ }^{13}$ which self-assemble at millimolar or sub-millimolar concentrations, most of the other LONs cac fall in the nanomolar range. Of note, the ratio of the probe molecules over the LON in the aggregates in such diluted solutions may somehow affect the value of the cac. Alternative techniques would therefore be highly desirable to confirm and shed light on the origin of these low cac numbers. Nevertheless, these investigations are further hampered by the tendency of the LONs to show complex aggregation patterns and kinetics. ${ }^{14}$ Recently, Langmuir-Blodgett films were prepared from the 11mer LON below (Figure 1). No stable monolayer at the air/water interface was observed probably because of the too short alkyl chains present in the structure of the LON. ${ }^{15}$ The LON molecules started to interact at a surface area of $1390 \AA^{2}$ per polar head somewhat ten times larger than traditional phospholipids. This phenomenon was ascribed to the much larger ON polar head. Furthermore, the compression and expansion of the film showed a hysteresis profile: reorganization at the air/water interface took place upon compression with the formation of multilayer structures with the LON. These later structures were indeed observed by atomic force microscopy.

The size of the oligonucleotide segment of the LON is one of the important parameters that governs the type of self-assembly formed. This has been investigated in some details by the group of Gianneschi. They were able to reversibly control the nature of the LON aggregates depending on the ON length. ${ }^{3,16}$ Shorter ON sequences of the LON favored vesicular aggregates. The latter were reversibly converted into micelles with longer ONs. The presence (or absence) of a spacer between the ON sequence and the lipidic segment of the LON was also shown to impact the morphology of the self-assembly to some extent, ${ }^{17}$ as well as the in vitro efficacy of si-RNA conjugates. ${ }^{18}$

On the other hand, the influence of other important parameters on the cac like (1) the base composition, (2) the presence of possible secondary structures in the $\mathrm{ON}$, and (3) the ionic strength has not been investigated so far. Indeed, secondary and to a lesser extent tertiary structures are possible in $\mathrm{ON}$ in general and always found in the structures of aptamers. Aptamers are small single stranded RNA or DNA molecules that can specifically recognize virtually any desired target. As such, they constitute a chemical equivalent of antibodies. The group of Tan combined the exquisite recognition specificity of aptamers with the self-aggregation/internatinalization properties of lipids to develop a LON aptamer capable of sensing ATP in living cells. ${ }^{19}$ Interestingly, these constructs, which showed no toxicity, were present as discrete molecules throughout the cytoplasm after self-internalization of the LON micelles. This greatly favored the detection of ATP in the cytoplasm.

Although not investigated in details in this article, the selfaggregation properties of the LONs may be at least in part responsible for the success of this design. Oligomerization of the aptamer molecules is indeed brought about by the micellar architecture. This setting is expected to lower the $\mathrm{k}_{\text {off }}$ and then prevent the dissociation of the target ATP from the complex.

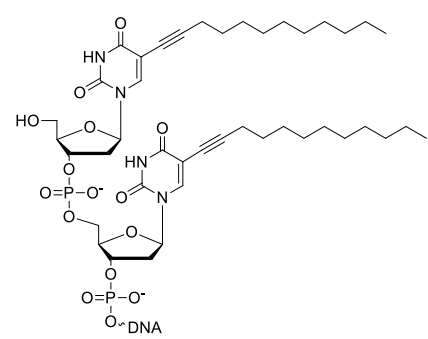

Figure 1. Example of a LON with short alkyl chains covalently linked to the nucleobases.

With an estimated 100 copies of aptamer molecules per micelle, the LON developed by Tan and coll. showed greatly enhanced recognition properties over the unmodified aptamer: the micelle aptamer still binds the targeted cells in the presence of blood, or at $37^{\circ} \mathrm{C}$ where the soft nature of the micelle proved far superior to the more conventional "hard" nanoparticle conjugates like gold or carbon nanotubes. ${ }^{2}$

Besides the "oligomerization" of the ON sequences that takes place at the surface of LON micelles, the sharing of the ions cloud between adjacent ONs, the so-called proximity effect, ${ }^{20}$ may also favor the affinity of the aptamer micelles for its target. Cations are actually required in the Stern layer (and above) around the nanoparticle to compensate for the anionic charges present in the ON sequences at the micellar surface. This phenomenon is entropically favorable as fewer cations are required for the neutralization of the same number of negative charges and/or the stabilization of secondary/tertiary $\mathrm{ON}$ structures. Similarly, the correct positioning of dodecyl alkyl chains in a DNA duplex was found to markedly increase the temperature of fusion of the duplex. ${ }^{21}$ ITC experiments showed that the favorable free energy of association between the 2 strands was primarily entropically driven as expected with a favorable hydrophobic effect.

LONs have also found applications in the field of DNA origami. The lipid segments of the LONs can be precisely positioned in these exquisite self-assembling 3-D DNA architectures. It was for instance possible to program the formation of discrete dimers of DNA-cubes through the specific aggregation of 2 DNA-cubes decorated with 4 LON molecules on one of their 6 faces. ${ }^{22}$ Interestingly the aggregation of the cubes took place at LON concentrations below their critical micellar concentration $(\mathrm{cmc})$.

Aggregates of LONs constitutes an ideal delivery vehicle for drug molecules in vivo: they are non toxic, they self-assemble into micellar structures which are dynamic and capable of hosting hydrophobic guests and the ON sequence can be used as a targeting agent and/or as a signal to trigger the release of the drug molecules. Our group took profit of these different properties to develop a LON-based delivery vehicle for paclitaxel molecules. ${ }^{23}$ Interestingly, the drug loading capabilities of the LON aggregates were found to depend not only on the nature of the lipid but also quite unexpectedly on the oligonucleotide sequence of the LON. Furthermore, binding 
of the complementary DNA to the LON aggregates led to the contraction of the micelles with the concomitant release of part of the drug molecules. Provided the ON sequence is found in the extracellular environment of the tumor cell, this platform could eventually find applications in the spatially controlled delivery of lipophilic drugs into malignant cells.

In a similar way, LON micelles where shown to induce capsid formation from the cowpea chlorotic mottle virus. ${ }^{24}$ The $28-\mathrm{nm}$ in length icosahedral virus particles were efficiently filled up with the LON micelles whose core can accommodate extra hydrophobic molecules like pyrene or others. These genomefree virus-like particles are very interesting in the context of the specific delivery of drug molecules to cells usually targeted by the virus.

\subsection{LONs incorporation into membranes}

If biotechnological applications of self-assembled LONs are rare in the literature due to their poor availability, they have found many interesting applications as tagging reagents for membranes, mostly self-assembled monolayers (SAM) or vesicles. In fact, DNA chips usually capitalize on "hard" covalent attachment of the ON to solid surfaces. In contrast, soft materials are dynamic in nature. ${ }^{25,26}$ They allow ON capture probes to rearrange and give homogeneous kinetics and thermodynamics upon annealing the target $\mathrm{ON} .{ }^{27}$ This property was for instance utilized for the reversible assembly of stacked nanodiscs decorated with LONs and with interdisc distances controlled by the length of the ON. ${ }^{28}$ Yet, one has to bear in mind that the adsorption of lipids into artificial or natural membranes is nothing but aspecific. Most of the lipidic membranes are heterogeneous in their lipidic content and distribution. Each lipid will therefore partition preferentially or specifically and with different kinetics into designated domains or rafts in the membrane. ${ }^{29}$ This has truly to be considered when LONs are used in a context of living cells. ${ }^{30,31}$ In addition, mammalian cells exhibit anionic sialic acid and glycosaminoglycans at their surface which were shown to disfavor LON partitioning due to repulsive electrostatic interactions. $^{32}$ As expected based on electrostatic considerations, shorter ON sequences were more favorably anchored in the membranes. Longer sequences have also a detrimental impact on the kinetics of fusion of the LON micelles with membranes, probably because of a greater distance between the lipids of the membrane and those of the micelles. ${ }^{2}$

Finally, LON self-assemble into aggregates whose dynamics have been poorly investigated. LON aggregates may well be kinetically and/or thermodynamically trapped with no possibility to partition into membranes. Clearly, the impact of the ON sequences in the free energy diagram of the LON aggregates and the residence time of each monomer in the aggregate are key elements that will necessitate deeper investigations in the future. The reader is referred to two recent review articles for other examples of surface functionalization with LONs. ${ }^{33,34}$

\section{Applications of LONs}

The group of Herrmann have designed a block copolymer LON: PFO-b-DNA (PFO = poly(9,9-di-n-octylfluorenyl-2,7diyl)) as a dispersing agent for single-wall Nanotubes (SWNT). This dispersant proved superior to other surfactants commonly employed. ${ }^{35}$ The semiconducting SWNT were selectively solubilized as individual entities from the crude nanotubes extract. Moreover, SWNT aqueous suspensions remained stable for more than 3 months. Very interestingly, these LONsolubilized semiconducting SWNT were used in the manufacture of a field-effect transistor by a pure self-assembly process controlled by the $\mathrm{ON}$ sequence in contrast to the tedious positioning of individual SWNT usually required in the manufacture of carbon nanotube electronics.

The ability of LONs to pair with complementary ONs and to be stably inserted into lipidic membranes has allowed their use as platforms for the detection of nucleic acids. Ideally, a powerful nucleic acid detection format should be able to directly detect sub-picomolar concentrations of DNA or RNA. As a result, nucleic acids are usually detected after some kind of preliminary amplification step (PCR or other), which is usually costly, time-consuming and certain sequences are more or less prone to being amplified by PCR. Signal amplification by any means is therefore necessary to avoid this amplification step and still detect low copy DNA or RNA molecules. The group of Höök developed an elegant strategy wherein LONs are inserted into vesicles with different lipid compositions. Provided the target $\mathrm{ON}$ sequence is present, it is sandwiched between the glass substrate and the vesicle decorated with the complementary sequence. ${ }^{36}$ Each single molecule target (A or $\mathrm{B}$ in Figure 2) binding event will translate into a huge number of co-lipid molecules (POPC or DPPC) immobilized on the surface thus amplifying the signal that can be detected by time of flight (TOF) secondary ion mass spectrometry (MS). Consequently, the MS signal for each co-lipid of the different vesicles is proportional to the concentration of each $\mathrm{ON}$ target sequence. 


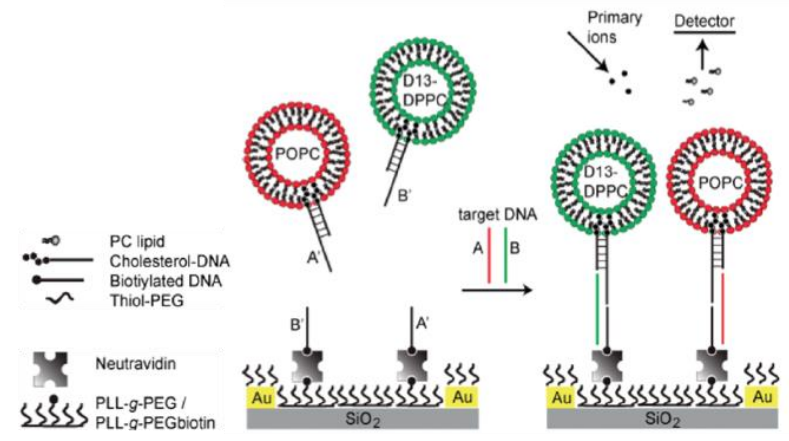

Figure 2. Example of signal amplification using vesicles encoded with LONs. In the presence of the complementary DNA targets (A or B) the vesicles are immobilized on the surface. This event allows an amplification of the number of secondary ions which are detected by mass spectrometry. (adapted from reference 36 ).

Based on a similar approach wherein the liposomes were loaded with a fluorescent dye, the same group was able to follow in real time the residence time of the vesicles once they are immobilized on the surface using total internal reflection fluorescence spectroscopy (TIRF). ${ }^{37}$ The immobilization of each single vesicle mediated by DNA duplex formation could be translated not only into the dissociation constant of the vesicle binding to the solid substrate but also into $\mathrm{k}_{\text {on }}$ and $\mathrm{k}_{\text {off. }}$. In fact, these kinetic parameters are far superior at discriminating between mutated DNA sequences compared to end-point thermodynamic measurements thus demonstrating the utility of this design for the detection of single point mutations.

Not only LONs are useful at detecting nucleic acid sequences, they can also serve as phase transfer agent for the purification of the complementary nucleic acid sequence. ${ }^{38}$ LON molecules reversibly partition between the water/organic solvent interface and reverse-micelles. Duplex formation between the LON and the water-soluble complementary DNA takes place at the interface. The targeted $\mathrm{ON}$ can therefore be extracted from water to the organic phase in a sequence-specific manner after the LONs form reverse-micelles in the organic phase.

Restoring the correct gene expression of cells by means of the administration of synthetic oligonucleotides constitutes the basis of gene therapy. Several ON-based drugs are now in

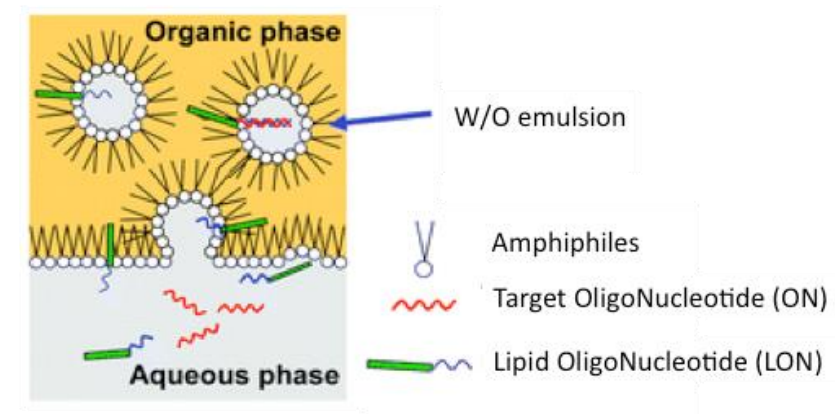

Figure 3. LONs (in green) associated with target ON (in red) can serve as phase transfer agent for the purification of the complementary nucleic acid sequence (adapted from reference 38 ). clinical trials or already marketed. For example, therapeutic progress has been achieved lately for patients with metastatic castration-resistant prostate cancer (mCRPC). Custirsen (OGX$011)$ is a second-generation 2'-methoxyethyl modified phosphorothioate antisense ON currently in phase III trials that inhibits expression of clusterin a cytoprotective chaperone protein that is upregulated in response to various anticancer therapies. Overexpression of clusterin inhibits apoptosis thus favouring malignant cell resistance to chemotherapies. ${ }^{39}$

MicroRNAs are natural genetic regulators of numerous cellular pathways and are involved in many diseases, including cancers. Several members of this class of small nucleic acid are currently emerging as new promising therapeutic targets and/or potentially relevant biomarkers. ${ }^{40}$ In that context, the LONQuantum Dot (QD) nanoplatform developed recently in our lab offers new opportunities as a dual diagnosis/therapeutic tool. ${ }^{41}$ Quantum dots were stably encapsulated with anti-miR21 LON molecules (Figure 4). miR-21 is a miRNA that is overexpressed in cancer cells. Surface plasmon resonance (SPR) studies indicate that just like in liposomes, ${ }^{42}$ the kinetics and thermodynamics of duplex formation at the surface of the QDs remain essentially unaffected compared to the same duplex free in solution. The LON-QDs were found to hybridize exclusively

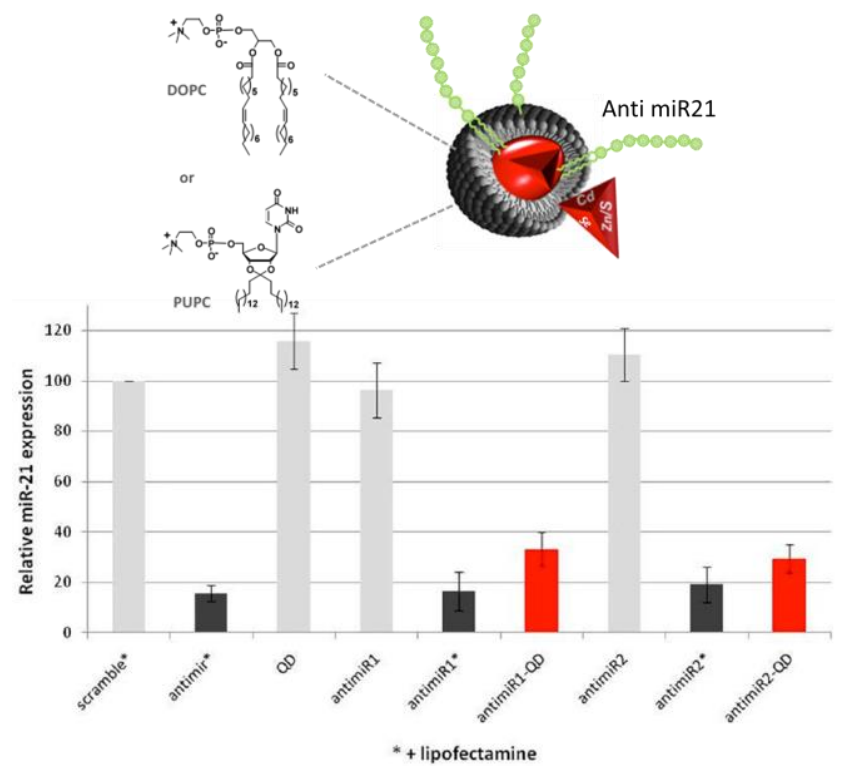

Figure 4. Up, schematic representation of a $\mathrm{LON}$ (in green) inserted in a membrane (in grey, DOPC or PUPC) that encapsulates the QD solid core (in red). Down, expression levels of miR21 in cancer cells incubated in different conditions, including control experiments. The platform inhibits the expression of miR21 (lines in red) of cancer cells in the absence of a transfecting reagent (lipofectamine) (adapted from reference 41).

with microRNA-21. This resulted in the down-regulation (30\%) of miR-21 expression in the absence of any tranfecting reagents.

As an alternative to LONs, fluorocarbon oligonucleotide conjugates (FONs) with either $\mathrm{C}_{8} \mathrm{~F}_{17}$ or $\mathrm{C}_{6} \mathrm{~F}_{13}$ hydrophobic and 
lipophobic chains were synthesized using a "post synthetic" grafting of the fluorocarbon moieties. ${ }^{43}$ Interestingly, it was demonstrated that these conjugates allows the cellular uptake of the ONs in different human cell lines, including hepatic Huh7, gastric epithelial NCI-N87 and embryonic kidney HEK293. These results suggest that FON aggregates may be used as a new tool for the delivery of oligonucleotides for intracellular targets, such as antisense sequences, aptamers or siRNA. Other examples of LON-based biomedical applications can be found in the literature. ${ }^{10}$

\section{Conclusion}

The numerous recent publications in the domain of hybrid lipid oligonucleotides indicate that these amphiphiles are promising tools in supramolecular chemistry as well as in biomedical applications. As witnessed lately by the burgeoning of new lipid oligonucleotide-based therapies targeting many different diseases, it is unquestionable that biomedical and biotechnological applications using these conjugates should greatly take advantage of the deeper understanding of their complex supramolecular chemistry. Consequently we are confident that this field will greatly evolve in the near future.

\section{Acknowledgements}

P.B. acknowledges financial support from the Army Research Office. This work has been supported by the French National Agency (ANR) in the frame of its programme Emergence (project TCTP-LASOCaP, ANR-11-EMMA-0022).

\section{Notes and references}

${ }^{a}$ INSERM U869, Bordeaux, F-33076, France.

${ }^{b}$ Université de Bordeaux, Bordeaux, F-33076, France.

\section{References:}

1. A. Patwa, A. Gissot, I. Bestel, and P. Barthélémy, Chem. Soc. Rev., 2011, 40, 5844-5854.

2. H. Liu, Z. Zhu, H. Kang, Y. Wu, K. Sefan, and W. Tan, Chem. Eur. J., 2010, 16, 3791-3797.

3. M. P. Thompson, M.-P. Chien, T.-H. Ku, A. M. Rush, and N. C. Gianneschi, Nano Lett., 2010, 10, 2690-2693.

4. A. Gissot, M. Camplo, M. W. Grinstaff, and P. Barthélémy, Org. Biomol. Chem., 2008, 6, 1324-1333.

5. M. Kwak and A. Herrmann, Chem. Soc. Rev., 2011, 40, 5745.

6. V. Moreau, E. Voirin, C. Paris, M. Kotera, M. Nothisen, J.-S. Rémy, J.-P. Behr, P. Erbacher, and N. Lenne-Samuel, Nucleic Acids Res., 2009, 37, e130

7. H. Lönnberg, Bioconjug. Chem., 2009, 20, 1065-1094.

8. Y. Singh, P. Murat, and E. Defrancq, Chem. Soc. Rev., 2010, 39 2054-2070.

9. R. L. Juliano, X. Ming, and O. Nakagawa, Acc. Chem. Res., 2012, 45, 1067-1076.

10. A. Patwa, A. Gissot, K. Oumzil, and P. Barthélémy, in DNA in Supramolecular Chemistry and Nanotechnology, E. Stulz and G. Clever, John Wiley \& Sons Ltd., 2014, p. in print.

11. T. Shibata, C. Dohno, and K. Nakatani, Chem. Commun., 2013, 49, $5501-5503$

12. L. Wang, Y. Feng, Z. Yang, Y.-M. He, Q.-H. Fan, and D. Liu, Chem. Commun., 2012, 48, 3715-3717.

13. K. Tanabe, Y. Ando, D. Hara, T. Ito, and S. Nishimoto, RSC Adv., 2014, 4, 13367-13370.
14. M. Banchelli, F. Betti, D. Berti, G. Caminati, F. B. Bombelli, T. Brown, L. M. Wilhelmsson, B. Nordén, and P. Baglioni, J. Phys. Chem. B, 2008, 112, 10942-10952.

15. R. Keller, M. Kwak, J. W. de Vries, C. Sawaryn, J. Wang, M. Anaya, K. Müllen, H.-J. Butt, A. Herrmann, and R. Berger, Colloids Surf. B Biointerfaces, 2013, 111, 439-445.

16. M.-P. Chien, A. M. Rush, M. P. Thompson, and N. C. Gianneschi, Angew. Chem. Int. Ed., 2010, 49, 5076-5080.

17. T. R. Pearce, B. Waybrant, and E. Kokkoli, Chem. Commun., 2014, 50, $210-212$

18. N. S. Petrova, I. V. Chernikov, M. I. Meschaninova, Ii. S. Dovydenko, A. G. Venyaminova, M. A. Zenkova, V. V. Vlassov, and E. L. Chernolovskaya, Nucleic Acids Res., 2012, 40, 2330-2344.

19. C. Wu, T. Chen, D. Han, M. You, L. Peng, S. Cansiz, G. Zhu, C. Li, X. Xiong, E. Jimenez, C. J. Yang, and W. Tan, ACS Nano, 2013, 7, 5724-5731.

20. I. Eryazici, T. R. Prytkova, G. C. Schatz, and S. T. Nguyen, J. Am. Chem. Soc., 2010, 132, 17068-17070.

21. C. Dohno, T. Shibata, M. Okazaki, S. Makishi, and K. Nakatani, Eur. J. Org. Chem., 2012, 2012, 5317-5323.

22. T. G. W. Edwardson, K. M. M. Carneiro, C. K. McLaughlin, C. J. Serpell, and H. F. Sleiman, Nat. Chem., 2013, 5, 868-875.

23. O. Pokholenko, A. Gissot, B. Vialet, K. Bathany, A. Thiéry, and P. Barthélémy, J. Mater. Chem. B, 2013, 1, 5329-5334.

24. M. Kwak, I. J. Minten, D.-M. Anaya, A. J. Musser, M. Brasch, R. J. M. Nolte, K. Müllen, J. J. L. M. Cornelissen, and A. Herrmann, J. Am. Chem. Soc., 2010, 132, 7834-7835.

25. A. Opdahl, D. Y. Petrovykh, H. Kimura-Suda, M. J. Tarlov, and L. J. Whitman, Proc. Natl. Acad. Sci., 2007, 104, 9-14.

26. M. Kwiat, R. Elnathan, M. Kwak, J. W. de Vries, A. Pevzner, Y. Engel, L. Burstein, A. Khatchtourints, A. Lichtenstein, E. Flaxer, A. Herrmann, and F. Patolsky, J. Am. Chem. Soc., 2012, 134, 280-292.

27. Y. Wu, K. Sefah, H. Liu, R. Wang, and W. Tan, Proc. Natl. Acad. Sci., 2010, 107, 5-10.

28. P. A. Beales, N. Geerts, K. K. Inampudi, H. Shigematsu, C. J. Wilson, and T. K. Vanderlick, J. Am. Chem. Soc., 2013, 135, 33353338 .

29. M. Loew, R. Springer, S. Scolari, F. Altenbrunn, O. Seitz, J. Liebscher, D. Huster, A. Herrmann, and A. Arbuzova, J. Am. Chem. Soc., 2010, 132, 16066-16072.

30. G. G. Borisenko, M. A. Zaitseva, A. N. Chuvilin, and G. E. Pozmogova, Nucleic Acids Res., 2009, 37, e28.

31. Z. J. Gartner and C. R. Bertozzi, Proc. Natl. Acad. Sci., 2009, 106, 4606-4610.

32. M. J. Palte and R. T. Raines, J. Am. Chem. Soc., 2012, 134, 62186223.

33. M. Schade, D. Berti, D. Huster, A. Herrmann, and A. Arbuzova, Adv. Colloid Interface Sci., 2014, 208, 235-251.

34. P. A. Beales and T. K. Vanderlick, Adv. Colloid Interface Sci., 2014, 207, 290-305.

35. M. Kwak, J. Gao, D. K. Prusty, A. J. Musser, V. A. Markov, N. Tombros, M. C. A. Stuart, W. R. Browne, E. J. Boekema, G. ten Brinke, H. T. Jonkman, B. J. van Wees, M. A. Loi, and A. Herrmann, Angew. Chem. Int. Ed., 2011, 50, 3206-3210.

36. A. Gunnarsson, P. Sjövall, and F. Höök, Nano Lett., 2010, 10, 732 737.

37. A. Gunnarsson, P. Jönsson, V. P. Zhdanov, and F. Höök, Nucleic Acids Res., 2009, 37, e99.

38. T. Maruyama, T. Hosogi, and M. Goto, Chem. Commun., 2007, 4450.

39. C. S. Higano, OncoTargets Ther., 2013, 6, 785-797.

40. E. Kim, J. Yang, J. Park, S. Kim, N. H. Kim, J. I. Yook, J.-S. Suh, S. Haam, and Y.-M. Huh, ACS Nano, 2012, 6, 8525-8535.

41. A. Aimé, N. Beztsinna, A. Patwa, A. Pokolenko, I. Bestel, and P. Barthélémy, Bioconjug. Chem., 2013, 24, 1345-1355.

42. A. Gissot, C. Di Primo, I. Bestel, G. Giannone, H. Chapuis, and P. Barthélémy, Chem. Commun., 2008, 5550-5552.

G. Godeau, H. Arnion, C. Brun, C. Staedel, and P. Barthélémy, MedChemComm, 2010, 1, 76-78. 